\title{
An oscilloscopic point-plotter interface for 8-bit microcomputers
}

\author{
GARY P. FINLEY and VINCENT DI LOLLO \\ University of Alberta, Edmonton, Alberta T6G 2E9, Canada
}

\begin{abstract}
An inexpensive interface capable of driving high-resolution point-plotting systems is described. Specific examples of modifications to the electronic hardware are detailed for the Rockwell Aim 65 , but the design is readily adaptable to most commercially available 8 -bit microcomputers. Some comparisons are drawn between salient features of raster-scan and of pointplotting display systems.
\end{abstract}

Microcomputers offer a range of control capabilities hitherto afforded only by more expensive minicomputer systems. Besides being inexpensive and readily available, microcomputers have the advantage of portability. This makes them eminently suitable for research projects that require subject populations (e.g., school children, institutionalized patients, and the elderly) whose transportation to an established laboratory is impractical or counterindicated.

Most commercially available microcomputers are equipped with video interfaces for displaying graphics and alphanumeric characters on a television monitor. Designed for interactive purposes, this type of display is inadequate for experimental applications requiring fine temporal or spatial resolution. Temporal resolution is tied to the period of the raster scan (approximately $16 \mathrm{msec}$ ), which is too coarse for many requirements (e.g., visual masking). In addition, the researcher is not given a choice of phosphors for special applications (e.g., fast-fading P15 phosphor). We surmounted these limitations and preserved the system's portability by designing and constructing an inexpensive interface capable of driving high-resolution oscilloscopic point plotters, such as the Tektronix 602 or the HewlettPackard 1333A.

\section{THE POINT-PLOTTING ELECTRONIC INTERFACE}

Patterns to be displayed may vary in complexity from sets of geometric figures or alphanumeric characters to a few points displayed at chosen locations on the screen. Regardless of complexity, all displays are made up of discrete points.

For each point that is to be plotted on the cathoderay tube (CRT), three coordinates corresponding to the horizontal position $(\mathrm{X})$, vertical position $(\mathrm{Y})$, and point intensity $(Z)$ are stored in the computer in digital form.

This work was supported by the Natural Sciences and Engineering Research Council of Canada, Grant A6592, to the second author. Requests for reprints should be sent to Gary P. Finley, Department of Psychology, Biological Sciences Building, University of Alberta, Edmonton, Alberta T6G 2E9, Canada.
In an 8-bit microcomputer, the data are stored in 8-bit digital words, each of which may represent any of $256\left(2^{8}\right)$ possible values. This range of values is adequate for a display of moderately high resolution if a single word is devoted to each of the spatial coordinates $X$ and $\mathrm{Y}$ and another to the point intensity $\mathrm{Z}$. Such a display can present any combination of 65,536 points arranged in a square matrix of 256 rows and columns. Each point in the matrix can be shown at any of 256 levels of intensity.

In plotting the display, the computer calculates, or retrieves from a table in its memory, three data words that define the coordinates of each point. These are presented sequentially to the plotting interface by the data bus or output port of the computer. The CRT display requires three corresponding analog voltage levels that simultaneously govern the plotting of one point. From this description of the point-plotting operation, three essential functions of the electronic interface may be identified: (1) the digital-to-analog (D/A) conversion of the coordinate data, (2) temporary storage of the sequential $\mathrm{X}, \mathrm{Y}$, and $\mathrm{Z}$ data in order that all three voltages may be provided to the CRT simultaneously, and (3) the provision of control signals for the required timing of the above functions.

The following will describe the hardware necessary to accomplish each of these three functions.

\section{CONVERTING DATA TO VOLTAGES}

The D/A conversion function is easily accomplished through the use of one of the several modular 8-bit D/A converters that are now commercially available. These require only the usual power supplies, plus a reference voltage supply and a few biasing components to convert 8-bit digital data into analog voltage levels. When operating an external load (such as the CRT display), most D/A converters require an output buffer in the form of an operational amplifier. This amplifier may also be used to scale the D/A converter output voltage by means of a simple gain adjustment. One special requirement is placed on the buffer amplifiers by 


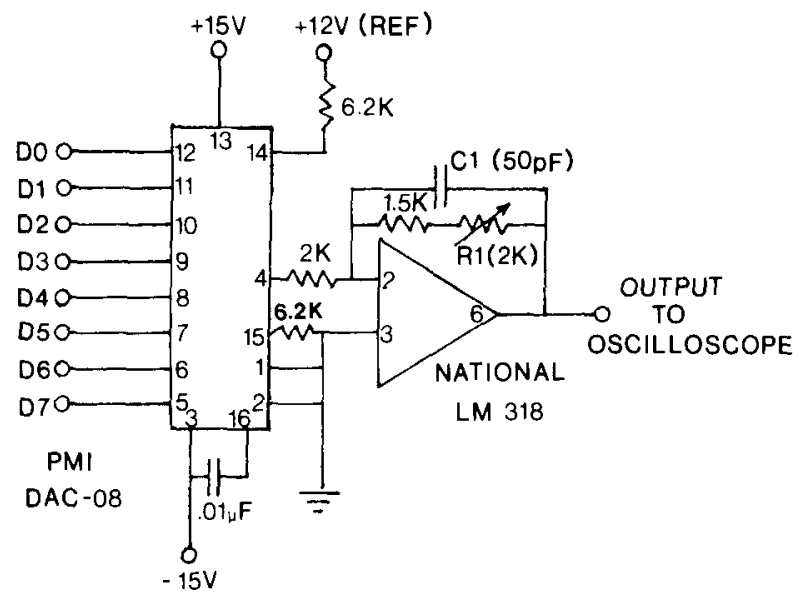

Figure 1. The 8 bits of digital data DO-D7 are converted to a voltage by a Precision Monolithics DAC-08 operating with a reference supply of $+12 \mathrm{~V}$. The resulting voltage is buffered by a National Semiconductor LM318 operational amplifier. The output is scaled by means of the gain adjustment $R 1$ until equal to $5 \mathrm{~V}$ when the input data are 11111111 binary (FF hexadecimal). The capacitor $\mathrm{Cl}$ serves to suppress ringing of the output voltage after rapid changes of level.

the high speed of modern microcomputers. The computer may be capable of plotting a point of the display every few microseconds, so the amplifiers are called upon to slew their output voltages very rapidly. For satisfactory performance, the amplifiers used should have an output voltage slew-rate specification in the region of $50-100 \mathrm{~V} /$ microsec.

Figure 1 shows the arrangement of $\mathrm{D} / \mathrm{A}$ converter and buffer amplifier components used in this example of an interface allowing a Rockwell Aim 65 microcomputer to perform X.Y plotting on a Tektronix 602 oscilloscope. The components for all three coordinates (X, $\mathrm{Y}$, and $\mathrm{Z}$ ) are identical, so only one set is drawn. The interface employs a circuit like the one shown for the conversion of the digital data into analog voltages for each of the three coordinates of a plotted point.

\section{LATCHING THE DATA}

The temporary storage of the $\mathrm{X}, \mathrm{Y}$, and $\mathrm{Z}$ data words is performed by three sets of 8-bit digital latches that are enabled by the control circuit at the times appropriate to the capture of the sequential $\mathrm{X}, \mathrm{Y}$, and $\mathrm{Z}$ coordinates. These data are held in the latches until new values are entered by the computer. In the cases of the $\mathrm{X}$ and $\mathrm{Y}$ channels, the latched data are continuously converted to analog voltages by the $\mathrm{X}$ and $\mathrm{Y} \mathrm{D} / \mathrm{A}$ converters. This arrangement is well suited to the rapid plotting of arrays of points, since an entire row of an array can be plotted by changing only the $X$ values, and an entire column by changing only the Ys.

The data from the computer's data bus should be buffered before connection to the data latches of the interface. The bus driving circuits of the 6502 micro- processor can operate up to five LS TTL load units. In computer systems that already contain external bus loads (like memory expansion boards or video controllers), the computer may not have the capacity to operate the three sets of latches in the plotting interface unless they obtain their data through a common bus buffer representing only one load unit. In this example, two 74LS00 circuits (with all eight gates connected as inverters) were used to perform the bus buffer function. The resulting inversion of the data is cancelled by connecting the inverting $(\overline{\mathrm{Q}})$ outputs of the data latches to the D/A converter inputs. In Figure 2 , the one set of bus buffers that serves all three coordinate channels is shown connected to the latches for one channel. An identical set of latches is required for each of the other two coordinate channels.

In the $\mathrm{Z}$ channel, additional circuitry is required to blank the CRT beam during each change of the $X$ and $Y$ coordinates. If this were not done, conspicuous "trails" would be seen to connect the displayed points as the CRT phosphor recorded the rapid passage of the beam from one location to another. Blanking of the beam is accomplished by the inclusion of a set of eight logic gates between the $Z$ data latches and the inputs to the $\mathrm{Z} \mathrm{D/A}$ converter. These gates hold the $\mathrm{Z} \mathrm{D} / \mathrm{A}$ converter inputs to logic zero unless enabled by a control signal. With all its inputs set to zeros, the Z D/A converter produces an output voltage near ground, and the application of this voltage to the $\mathrm{Z}$-axis input of the CRT blanks the beam. When a point is to be plotted, a control signal from the computer enables a multivibrator

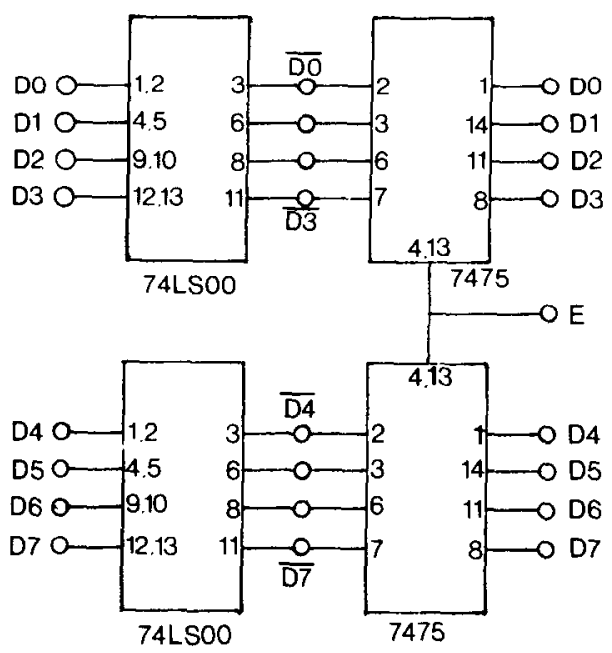

Figure 2. Two $74 \mathrm{LS} 00$ circuits connected as inverting buffers deliver data to the pair of 7475 latches for one channel. The latch enable signal for each channel $(E)$ is provided by the inverse of the address decoder output for that channel (see Figure 4). A single 8-bit latch circuit (such as the 74100 or 74273) can be used in place of the two 4-bit devices noted, resulting in a reduction of the component count of the plotting interface. However, the 8-bit devices are less commonly available than the $7475 \mathrm{~s}$ used here. 


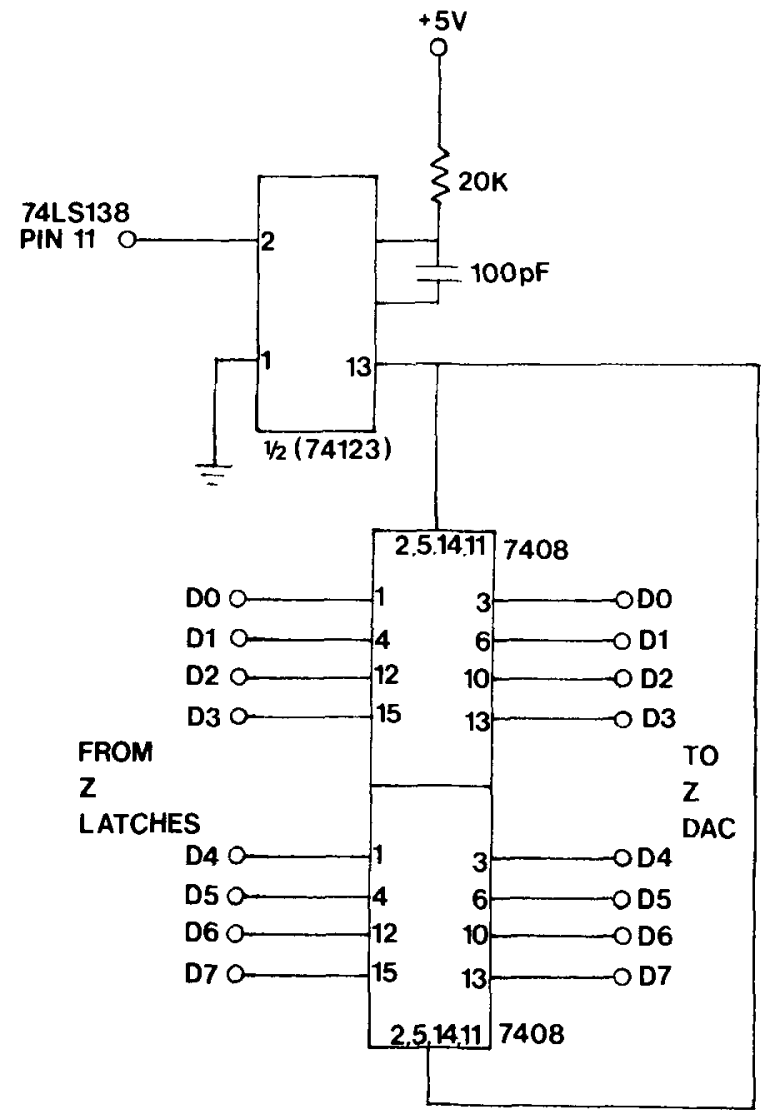

Figure 3. When triggered by the address decoder output (see Figure 4) the 74123 monostable produces a positive logic pulse of 1 microsec duration. During this pulse, the two 7408 circuits pass the 8 bits of the latched $Z$ data to the $Z$ DAC circuit. At other times the $Z$ DAC inputs are all held to the logic zero condition.

circuit in the interface that produces a positive logic pulse of known duration. This pulse allows the logic gates to pass the previously latched $Z$ data to the $Z$ $\mathrm{D} / \mathrm{A}$ converter inputs. The $\mathrm{Z} \mathrm{D} / \mathrm{A}$ converter output voltage thus assumes a value appropriate to the latched $\mathrm{Z}$ data only for the duration of the enabling pulse from the multivibrator. This causes the CRT beam to have the desired intensity for the known duration of the pulse (around 1 microsec), after which it will be blanked again in preparation for the next change of location. The multivibrator and $\mathrm{Z}$ data logic gates for the beam unblanking function are seen in Figure 3.

\section{TIMING AND CONTROL}

From the above description of the data-latching and beam-blanking functions, it can be seen that four sequential operations are required for the plotting of an isolated dot at some arbitrary location. These are the latching of the data for the $\mathrm{X}, \mathrm{Y}$, and $\mathrm{Z}$ coordinates in turn, and, finally, the unblanking of the beam for presentation of the dot. In this design, the generation of a control pulse appropriate to each operation is governed by a digital address decoder. This decoder makes it possible for the programmer to treat each operation as a fictitious address in the computer's memory, somewhere outside of the address range actually used by the computer. As each coordinate data word is generated in the program, it is stored to the memory address assigned to the appropriate set of data latches in the interface. The address decoder in the interface then issues a pulse that causes the word to be held in the latches for conversion to an analog voltage by the D/A converter for that channel. In the case of the beam unblanking function, the data word stored to the unblanking address is irrelevant, since there are no latches to store it. However, the act of storing any data word to the unblanking address causes the address decoder to trigger the multivibrator that governs the unblanking of the CRT beam.

Figure 4 shows the address decoder used to enable sequentially the $\mathrm{X}, \mathrm{Y}$, and $\mathrm{Z}$ data latches and the beam unblanking monostable under the control of the point plotting program. The signals $\mathrm{A} 0, \mathrm{~A} 1$, and $\mathrm{A} 2$ are the three lowest order lines of the Aim 65 address bus. CS-9 is a signal generated by the computer whenever addresses $9000-9$ FFF (hexidecimal) are active. $\phi 2$ is the second phase of the two-phase clock pulse of the 6502 microprocessor, and $\mathrm{R} / \overline{\mathrm{W}}$ is a signal indicating whether the computer is writing to or reading from a memory address. When connected as shown to these control and address signals of the Aim 65, four of the address decoders' outputs will be enabled by the addresses 9001-9004. The functions below are thus activated when the computer writes data to the memory addressess shown:

\begin{tabular}{cl} 
Address (Hex) & \multicolumn{1}{c}{ Function } \\
\hline 9001 & X data latch enable \\
9002 & Y data latch enable \\
9003 & Z data latch enable \\
9004 & beam unblank
\end{tabular}

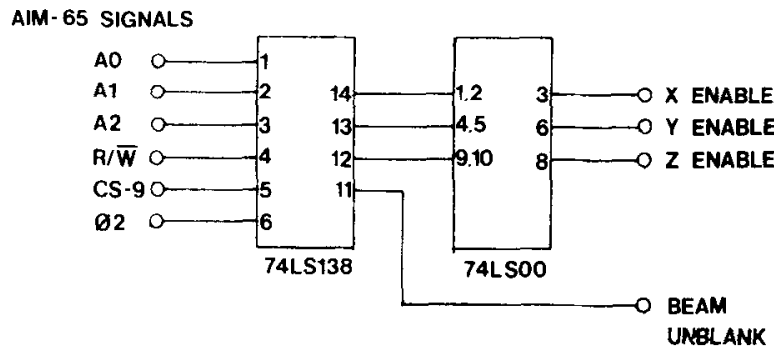

Figure 4. Six Aim-65 address and control signals are decoded by a $74 \mathrm{LS} 138$ decoder to activate the four plotting functions under a sequential control of the plotting program. The decoder outputs for the latch enables are inverted by gates in a 74LSOO circuit to obtain the logic state required by the 7475 data latches. 
Table 1

Program to Plot the Corners of a Square in Counterclockwise Order from the Lower Left

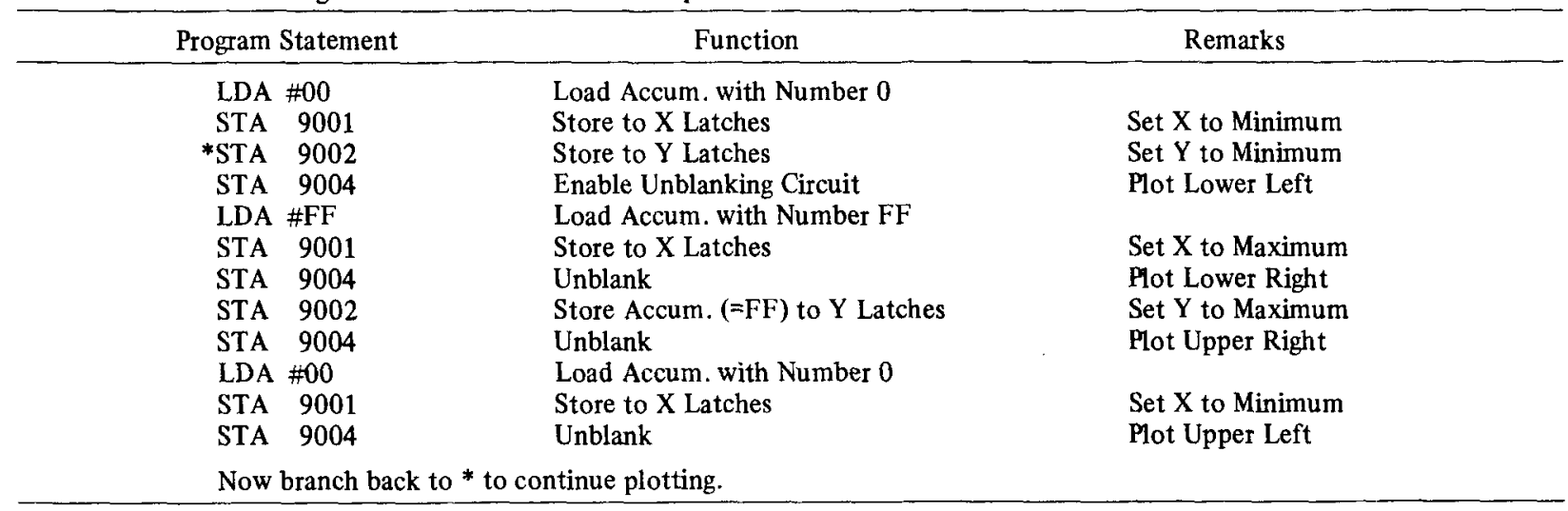

\section{GENERAL COMMENTS}

Many visual experiments in psychology can be performed satisfactorally on video TV monitors. Recently, Reed (1979) described a variety of techniques that permit a computer-controlled video display unit to function as a multifield tachistoscope. Reed also showed how, with some simple modifications to the computer's electronic hardware, it is possible to produce video displays of durations as brief as $1 \mathrm{msec}$. However, such modifications also result in proportional reduction in the size of the viewing area. In effect, the temporal resolution of a whole-field video system is tied to the period of the raster scan. This means that such important temporal parameters as exposure duration or interstimulus interval can vary only in steps of approximately $16 \mathrm{msec}$ for sequential displays shown at about the same location on the screen.

In general, as was pointed out by Reed (1979), relatively inexpensive video systems are clearly to be favored over more expensive point-plotting display systems if fine temporal resolution and type of phosphor are not concerns. The interface described above is intended for use in experiments in which the spatiotemporal constraints of video systems are unacceptable and the employment of oscilloscopic point plotters becomes necessary. The hardware requirements are easy to implement, are relatively inexpensive, and maintain the portability of the system. Notably, the design of the interface does not interfere with any existing video facilities on the computer board, thus providing a choice between video and point-plotting display modes. It should also be noted that the present design makes no demand on the computer's digital input-output (I/O) facilities. Indeed, an alternative method of performing the same function would utilize the computer's $I / 0$ ports for transferring data to each D/A converter and for addressing the three separate devices. However, besides preempting the use of at least 10 bits of $\mathrm{I} / \mathrm{O}$ ( 8 bits of data and 2 bits for device addressing), this method would not result in greater ease of programming or in higher transfer rates. A sample program to plot four dots, one at each corner of the viewing surface, is shown in Table 1.

The rate of plotting of the dots varies with the software requirements of the pattern to be displayed. In the example illustrated in Table 1, one dot was plotted every 9.4 microsec, corresponding to a plotting rate of approximately $100 \mathrm{kHz}$. In the worst case, in which counters are to be set up and branching decisions are to be made, one dot can be plotted every 27 microsec, corresponding to a plotting rate of approximately $37 \mathrm{kHz}$.

\section{REFERENCE}

REed, A. V. Microcomputer display timing: Problems and solutions. Behavior Research Methods \& Instrumentation, 1979, 11, 572-576.

(Received for publication August 25, 1980; revision accepted November 17,1980 .) 